\title{
Análisis de tiempos de espera en pacientes con cáncer de cabeza y cuello en el Hospital San Juan de Dios
}

\author{
Analysis of wait time in head and neck cancer in the San Juan de Dios Hospital
}

Gerardo Oberreuter $\mathbf{E}^{\mathbf{1}}$, Carolina Oliva G² , José Miguel Contreras $\mathbf{R}^{\mathbf{1}}$, Felipe Cardemil $\mathbf{M}^{\mathbf{1}}$.

\section{RESUMEN}

Introducción: Los cánceres de cabeza y cuello (CCyC) son reconocibles precozmente mediante la clínica y exámenes poco invasivo, sin embargo, existe importante demora entre las primeras molestias del paciente y el tratamiento.

objetivo: Describir los tiempos involucrados entre los primeros síntomas y el tratamiento.

Material y método: Estudio descriptivo retrospectivo, mediante evaluación de fichas de pacientes diagnosticados y tratados por CCyC en otorrinolaringología del Hospital San Juan de Dios durante 18 meses.

Resultados: Fueron elegibles 33 casos. El 93,7\% correspondieron a carcinomas escamosos. Fueron comprometidos principalmente laringe $(45,4 \%)$ y orofaringe $(24,2 \%)$. El 90,2\% se diagnosticó en estadio avanzado.

Los pacientes demoraron 17 semanas en consultar desde sus primeros síntomas. Pasó una semana para ser evaluados por el especialista. Este tardó una semana en obtener una biopsia. El resultado histológico tomó tres semanas y el tratamiento se realizó a las cinco semanas. Quienes recibieron cirugía como primer tratamiento tardaron cuatro semanas en recibirla. Quienes fueron a quimiorradioterapia como coadyuvancia esperaron 11,5 semanas. Mientras, quienes recibieron quimiorradioterapia como único tratamiento, tardaron 8 semanas.

Conclusión: Los principales retrasos en el manejo de estos pacientes son: la demora del paciente en consultar, el informe histológico y el acceso al tratamiento al conocerse el diagnóstico, especialmente cuando el tratamiento es la radio-quimioterapia.

Palabras clave: Cáncer, cabeza y cuello, tiempo, demora, tratamiento.

\begin{abstract}
Introduction: Head and neck cancers (HNC) are recognizable by clinical and early minimally invasive tests, however, there is significant delay between the first symptoms and treatment.
\end{abstract}

1 Servicio de Otorrinolaringología, Hospital San Juan de Dios - Facultad de Medicina, Universidad de Chile.

2 Interna de Medicina. Hospital San Juan de Dios. Universidad de Chile.

Recibido el 24 de noviembre, 2016. Aceptado el 18 de diciembre, 2016. 
Aim: To describe the times involved between the first symptoms and treatment.

Material and method: Descriptive and retrospective study, by evaluating records of patients diagnosed and treated for HNC in otolaryngology at the Hospital San Juan de Dios, for 18 months.

Results: 33 cases were eligible. 93.7\% corresponded to squamous cell carcinoma. The sites most committed were larynx (45.4\%) and (24.2\%); the diagnosis was made in advanced stages in $90.2 \%$ of the cases.

The patients took 17 weeks to consult since their first symptoms. It took 1 week to be evaluated by the specialist. It took 1 week to get a biopsy. The histological result took 3 weeks and treatment was performed at 5 weeks. Who received surgery as first treatment took 4 weeks to receive it. Who went to chemo-radiotherapy as co-adjuvant waited 11.5 weeks. Those receiving chemo-radiotherapy as the only treatment, 8 weeks.

Conclusion: The major delays are the patient delay in consulting, the time delay in the histological report and access to treatment when knowing the diagnosis, especially when treatment is radio-chemotherapy.

Key words: Cancer, head and neck, time, delay, treatment.

\section{INTRODUCCIÓN}

Los tumores de cabeza y cuello son posibles de diagnosticar mediante la evaluación de síntomas, signos y exámenes poco invasivos. Para hallarlos precozmente debe existir un alto grado de sospecha por el examinador y una consulta oportuna por parte del paciente, sin embargo, la mayoría de las veces esto no ocurre. Con respecto a la epidemiología nacional, en Chile se dispone de registros poblacionales de cáncer acreditados por la International Agency for Research on Cancer de la Organización Mundial de la Salud, en Valdivia, Antofagasta y Biobío ${ }^{1}$. Se ha estimado que en Chile la incidencia de cáncer de laringe es 1,2 casos por cada 100.000 habitantes, de labio y cavidad oral es 1,3 casos por cada 100.000 habitantes, $y$ de orofaringe e hipofaringe es 0,5 casos por cada 100.000 habitantes $^{2}$. Entre el $90 \%$ y $95 \%$ corresponden a carcinomas de células escamosas, presentándose más de la mitad de ellos localmente avanzados al momento del diagnóstico ${ }^{3}$. A pesar de los avances en el tratamiento, la supervivencia no ha mejorado durante las últimas tres décadas, siendo un factor importante en la explicación de este hecho la demora en el diagnóstico ${ }^{4}$. El pronóstico en estadios avanzados es peor que en los tumores diagnosticados en etapas precoces y, asimismo, que el retraso en el proceso diagnóstico se asocia a necesidad de realizar tratamientos más complejos en estadios más avanzados ${ }^{5}$. La tasa de supervivencia a 5 años para los pacientes con enfermedad localizada es del $82 \%$, la de los pacientes diagnosticados con enfermedad regional es $51 \%$ y la de los pacientes con enfermedad diseminada sólo de $27,6 \%$. Hay varios factores que han sido evaluados como marcadores pronósticos independientes para el cáncer de cabeza y cuello, identificándose la etapa del tumor al momento del diagnóstico como el más importante ${ }^{6}$. Este retraso se refiere al período de tiempo entre la aparición de los primeros síntomas hasta que se establece el diagnóstico. A grandes rasgos, se puede dividir en dos fases: el período comprendido entre el inicio de los síntomas hasta la búsqueda de atención médica (retraso del paciente) y el tiempo entre la primera consulta con un profesional de la salud y la confirmación histopatológica (retraso del sistema de salud) $)^{7,8}$. Mientras el retraso profesional puede medirse con relativa precisión, el retraso del paciente tiende a mostrar errores en su evaluación, ya que depende únicamente de su percepción ${ }^{9}$. Una vez obtenido el diagnóstico, es necesario definir y realizar el mejor tratamiento para cada paciente, lo cual también involucra etapas sucesivas con demoras entre una y otra. Estos períodos del proceso diagnóstico-terapéutico son divisibles en varias "subetapas", lo que permite orientar acerca de las falencias en el sistema de salud. De esta forma se hace posible trabajar adecuadamente en él. A pesar de esto, no existen publicaciones en Chile que analicen los tiempos recién descritos. 


\section{OBJETIVO}

El objetivo principal es describir los períodos de tiempo involucrados entre los primeros síntomas hasta el tratamiento, incluyendo la espera según tipo de tratamiento recibido, en los pacientes portadores de cáncer de cabeza y cuello en el Hospital San Juan de Dios entre los años 2015 y 2016. Los objetivos secundarios son describir las características demográficas de estos pacientes, los sitios comprometidos y los estadios al momento del diagnóstico.

\section{MATERIAL Y MÉTODO}

Se realizó un estudio retrospectivo, mediante el análisis de fichas de pacientes con diagnóstico y tratamiento de cáncer de cabeza y cuello en el Servicio de Otorrinolaringología del Hospital San Juan de Dios entre el 1 de abril de 2015 y el 1 de octubre de 2016 (18 meses). Los pacientes correspondientes a recidivas de cáncer fueron excluidos, por no presentar características similares en cuanto a su evolución sintomática y por ya estar en controles con un médico especialista. De las fichas se recolectaron: sexo, edad (en años), diagnóstico según estudio de biopsia diferida por anatomía patológica, clasificación según los estadios de la séptima edición de la American Joint Committee on Cancer (AJCC), tratamiento recibido (cirugía, quimioterapia y/o radioterapia como tratamiento inicial 0 en adyuvancia).

Con respecto al tiempo transcurrido entre las primeras molestias del enfermo hasta que se realizó el primer tratamiento en él, se dividió de la siguiente manera:

- Intervalo de tiempo en semanas entre la primera molestia atribuible al cáncer y la primera consulta con un profesional de la salud (odontólogo, médico general o médico otorrinolaringólogo).

- Intervalo de tiempo en semanas entre la primera consulta con un profesional de la salud y la derivación a médico otorrinolaringólogo (evaluado como la primera consulta con éste).

- Intervalo de tiempo en semanas entre la primera consulta con el médico otorrinolaringólogo y la toma de biopsia (se consideró la toma de biopsia que logró dar con el diagnóstico).

- Intervalo de tiempo en semanas entre la toma de biopsia y el informe de anatomía patológica.

- Intervalo de tiempo en semanas entre el informe de anatomía patológica y el inicio del primer tratamiento (cirugía, quimioterapia y/o radioterapia neoadyuvantes)

- Intervalo de tiempo en semanas entre la cirugía (para quienes éste fue su primer tratamiento) y el inicio de quimio/radioterapia adyuvante, cuando ésta fue indicada y aprobada por un comité de oncología.

\section{RESULTADOS}

Se encontraron 47 casos que cumplían con los criterios de ingreso para el estudio. De éstas, 12 no fueron incluidas por tener información incompleta por estar aún en proceso diagnóstico y/o terapéutico. Otras dos fueron excluidas por corresponder a recidivas tumorales, pues son pacientes que ya están en control con médico especialista y no representan consultas espontáneas. Se consideraron para el estudio 33 casos.

De ellos, el 78,9\% corresponden a hombres entre 24 a 86 años, promediando 65 años. Los sitios primarios comprometidos fueron, en orden de frecuencia: laringe $45,4 \%$; orofaringe $24,2 \%$; hipofaringe $12,1 \%$; cavidad oral $6 \%$; cavidades paranasales $6 \%$ (todos carcinomas escamosos), un caso de condrosarcoma de hioides $3,15 \%$ y un adenoideo-quístico submandibular 3,15\%.

Considerando sólo los carcinomas de células escamosas, al momento del diagnóstico se presentaron en estadio: IVA 54,8\%, III 19,3\%, IVB 9,7\%, IVC $6,4 \%$, II $6,4 \%$ y I $3,4 \%$.

Con respecto a los tiempos transcurridos entre las distintas etapas del diagnóstico y el tratamiento, se obtuvieron los siguientes resultados, presentados en medianas de tiempos en semanas y entre paréntesis el rango de tiempo: Primer síntoma - Primera consulta con un profesional de la salud: 17 semanas (6-312). Primera consulta - Derivación al médico especialista: 1 semana (1-68). Desde la primera consulta con el especialista - Toma de biopsia: 1 semana (1-28). Biopsia - Informe 
histopatológico, 3 semanas (1-7). Con respecto al tratamiento, se realizó una división en los tiempos según la terapia recibida. Aquellos pacientes que recibieron cirugía como primer tratamiento tardaron 4 semanas en recibirla (rango 1-16). Mientras, quienes fueron a quimio y/0 radioterapia como coadyuvancia esperaron 11,5 semanas (rango 8-20 semanas. Aquellos que recibieron quimiorradioterapia como único tratamiento, tardaron 8 semanas (rango 3-12 semanas).

\section{DISCUSIÓN}

Los datos demográficos del presente estudio, en cuanto a edad y sexo, son similares a lo encontrado en la literatura. Lo mismo ocurre con la distribución del sitio de origen del tumor ${ }^{3,10}$. Cabe destacar que el $90,2 \%$ de nuestros pacientes se presentaron en estadio avanzado (estadio III en adelante), lo cual supera considerablemente el 60\%-70\% reportado en experiencias internacionales ${ }^{11,12}$. Esto tiene múltiples explicaciones, siendo una de ellas, sin duda, la demora en el diagnóstico. Dado el patrón de progresión lento de la patología tumoral, es probable que los pacientes se adapten a las molestias iniciales y sólo consulten cuando éstas sean más severas (p.ej. disnea o disfagia severa), lo que se traduce en tumores más avanzados ${ }^{13}$. Esto se condice con los 4 meses que existen, como mediana, entre el inicio de las molestias del paciente y su primera consulta. Sin embargo, no podemos obviar las dificultades para acceder a una consulta médica, especialmente difícil para la población que atiende el Hospital San Juan de Dios, que en gran parte corresponde a un nivel socioeconómico bajo: Faltar un día al trabajo, costear el transporte, copagos por la atención, y otros, lo que suma algunos días o semanas de demora a la consulta con algún profesional de la salud. Esta parte del retraso en el diagnóstico va más allá del sistema de salud (pero sin excluirlo) e involucra al sistema de salud en su totalidad. En esta línea, la educación podría jugar un rol importante, ya que fumar y el consumo de alcohol son factores de riesgos frecuentes y conocidos por la población en general como gatillantes de distintas condiciones médicas. Se ha visto que concientizar a las personas con estos factores de riesgo a buscar ayuda médica después de los síntomas iniciales sugerentes de un cáncer de cabeza y cuello, favorece su pronóstico ${ }^{14}$.

Con respecto al tiempo entre la primera consulta con un profesional de la salud y la derivación al especialista, la mediana fue de una semana. Sin embargo, el rango de tiempo fue de 1 a 68 semanas. En el otro extremo, en los casos más prolongados, hubo dos situaciones en general: casos compatibles con cuadros benignos que no producían alarma en el médico no especialista (p.ej. otalgias intermitentes en tumores de seno piriforme) y otros casos donde simplemente faltó un grado más alto de sospecha. Aquí también se detectaron problemas de tipo administrativo, como derivar pacientes de forma no-urgente con sospecha de cáncer, siendo citados meses después de la derivación. Estos dos últimos escenarios podrían manejarse con educación continua en la atención primaria en salud.

Una vez evaluado el paciente por el especialista, la toma de biopsia tardó una semana como mediana, con entre 0-28 semanas de rango. Cero semanas correspondió a situaciones donde existía una alta sospecha y posibilidad de toma de biopsia en el box de atención (p.ej. tumores de cavidad oral). Los casos de larga espera fueron principalmente por la necesidad de un pabellón para tomar la muestra. Esto implica: obtener un pabellón en un sistema con alta lista de espera para ellos; estabilizar sus comorbilidades a pacientes con edad avanzada, sedentarios, con tabaquismo y consumo de alcohol importante, etc.

Obtenida la biopsia, la mediana para el informe histológico fue de tres semanas, con 1 a 7 semanas de rango. Los carcinomas escamosos de cabeza y cuello en adultos no corresponden a ninguna patología incluida en el programa de Garantías Explícitas en Salud (GES) ${ }^{15}$. Dadas las obligaciones e implicancias económicas que este programa significan para nuestro hospital, los servicios de anatomía patológica se enfocan en analizar muestras de estos problemas de salud, retrasando el informe definitivo de nuestros pacientes.

Con respecto al tratamiento, quisimos hacer la diferencia entre el tiempo requerido para obtener una cirugía y obtener quimiorradioterapia. Encontramos una mediana de 4 semanas para el primero y de 8 para el segundo. Las razones de la 
tardanza para realizar la cirugía una vez obtenido el informe histológico son similares a las descritas para la toma de biopsia en pabellón, a lo que se debe agregar la necesidad de poder acceder a un pabellón que permita más horas de cirugía.

Finalmente, sobre la quimiorradioterapia, la principal razón de los más de 2 meses de espera para acceder a ella una vez indicada como tratamiento primario y casi 3 meses en el caso de coadyuvancia, es la dificultad para acceder a este recurso. Si bien en algunos casos existió retraso por la necesidad de estabilizar las comorbilidades de los enfermos, o su recuperación posquirúrgica, la mayor parte de las veces la espera fue sólo por falta de disponibilidad del recurso. Esta situación es preocupante, pues se ha visto en cáncer de cabeza y cuello, en dos grandes estudios, un aumento significativo en la tasa de recidiva local si la radioterapia se retrasa, especialmente más de 40 días ${ }^{16,17}$. A pesar de todos los esfuerzos y estudios que se han hecho con respecto a la conservación de órganos en estos tumores, su utilidad se ve cuestionada si el tratamiento no puede ser realizado oportunamente. Por lo tanto, como equipo nos parece que esto es algo que se debe considerar a la hora de evaluar un tratamiento de órgano de preservación con radioterapia o radioquimioterapia como tratamiento inicial, en la medida que lamen-

\section{BIBLIOGRAFÍA}

1. Cardemil F. Epidemiología del carcinoma escamoso de cabeza y cuello. Rev Chil Cir 2014; 66(6): 614-20.

2. World Health Organization (WHO). GLOBOCAN, International Agency for Research on Cancer (IARC). 2014. (Disponible en http://globocan. iarc.fr Consultado el 14 de Septiembre del 2016.)

3. Jemal $A$, Siegel $R, X u$ J, et al. Cancer Statistics. CA Cancer J Clin 2010; 60: 277-300.

4. Gómez I, Seoane J, Varela-Centelles P, Diz P, TAккочCHE $B$. Is diagnostic delay related to advanced stage oral cancer? A meta-analysis. Eur J Oral Sci 2009; 117(5): 541-6.

5. Kowalski LP, CaRVaLho AL. Influence of time delay and clinical upstaging in the prognosis of head and neck cancer. Oral Oncol 2001; 37: 94-8. tablemente no siempre tenemos acceso oportuno al recurso, lo que podría tener un impacto negativo en el pronóstico de los pacientes con cáncer. Es uno de los tantos motivos por los cuales terapias que puedan realizarse más oportunamente, como las estrategias quirúrgicas, deberían seguir siendo consideradas como una opción terapéutica válida en estos casos.

\section{CONCLUSIÓN}

Existe una importante demora entre los primeros síntomas del paciente hasta que éste comienza su tratamiento, donde destaca la tardanza en realizar la primera consulta. Llama la atención también la demora en el inicio de la quimiorradioterapia una vez que ésta fue indicada. Todas estas semanas de tratamiento perdido podrían influir negativamente en el pronóstico de estos pacientes, y es responsabilidad tanto del sistema de salud como de los médicos en general trabajar en mejorar estos tiempos. En este contexto, es necesario aumentar la cantidad de publicaciones con estas experiencias a nivel nacional para así tener herramientas de comparación y análisis para saber dónde enfocar los esfuerzos y recursos para abordar este problema.

6. Seoane J, Takkouche B, Varela-Centelles P, Tomás I, Seoane-Romero JM. Impact of delay in diagnosis on survival to head and neck carcinomas: a systematic review with meta-analysis. Clin Otolaryngol 2012; 37(2): 99-106.

7. Mackillop WJ, Bates JH, O'Sullivan B, Withers HR. The effect of delay in treatment on local control by radiotherapy. Int J Radiat Oncol Biol Phys 1996; 34: 243-50.

8. Allison P, Locker D, Feine JS. The role of diagnostic delays in the prognosis of oral cancer: a review of the literature. Oral Oncol 1998; 34 : 161-70.

9. Carvalho Al, Pintos J, Schlecht NF, Oliveira BV, Fava AS, Curado MP, et al. Predictive factors for diagnosis of advanced-stage squamous cell carcinoma ofthe head and neck. Arch Otolaryngol Head Neck Surg 2002; 128: 313-8. 
10. Carvalho Al, Pintos J, Schlecht NF, Oliveira BV, Fava AS, Curado MP, et al. Predictive factors for diagnosis of advanced-stage squamous cell carcinoma ofthe head and neck. Arch Otolaryngol Head Neck Surg 2002; 128: 313-8.

11. Kaufman S, Grabau JC, Lore J. Symptomatology in head and neck cancer: a quantitative review of 385 cases. Am J Public Health 1980; 70: 520-2.

12. Dhooge IJ, Albers FW, van Caumenberge PB. Clinical characteristics and diagnostic delay of head and neck cancer: results from a prospective study in Belgium. Eur J Surg Oncol 1996; 22: 354-8.

13. Tromp DM, Brouha XD, HordiJk GJ, WinnuBSt JA, DE LEEUW JR. Patient factors associated with delay in primary care among patients with head and neck carcinoma: a case-series analysis. Fam Pract 2005; 22: 554-9.
14. Goy J, Hall SF, Feldman-Stewart D, Groome PA. Diagnostic delay and disease stage in head and neck cancer: a systematic review. Laryngoscope 2009; 119: 889-98.

15. Ministerio de Salud de Chile. Garantías Explícitas en Salud. Disponible en http: //diprece.minsal. cl/le-informamos/auge/. Consultado el 14 de septiembre de 2016.

16. Fortin A, Bairati I, Albert M, Moore L, Allard J, Couture $C$. Effect of treatment delay on outcome of patients with early-stage head-and-neck carcinoma receiving radical radiotherapy. Int $\mathrm{J}$ Radiat Oncol Biol Phys 2002; 52: 929-36.

17. Waaluer A, Terhaard CH, Dehnad H, HordiJk GJ, Van Leeuwen MS, RaAymakers CP, LagendiJik JJ. Waiting times for radiotherapy: consequences of volume increase for the TCP in oropharyngeal carcinoma. Radiother Oncol 2003; 66(3): 271-6.

Dirección: Felipe Cardemil M.

Servicio de Otorrinolaringología, Hospital San Juan de Dios - Universidad de Chile

E mail: felipecardemil@med.uchile.cl 